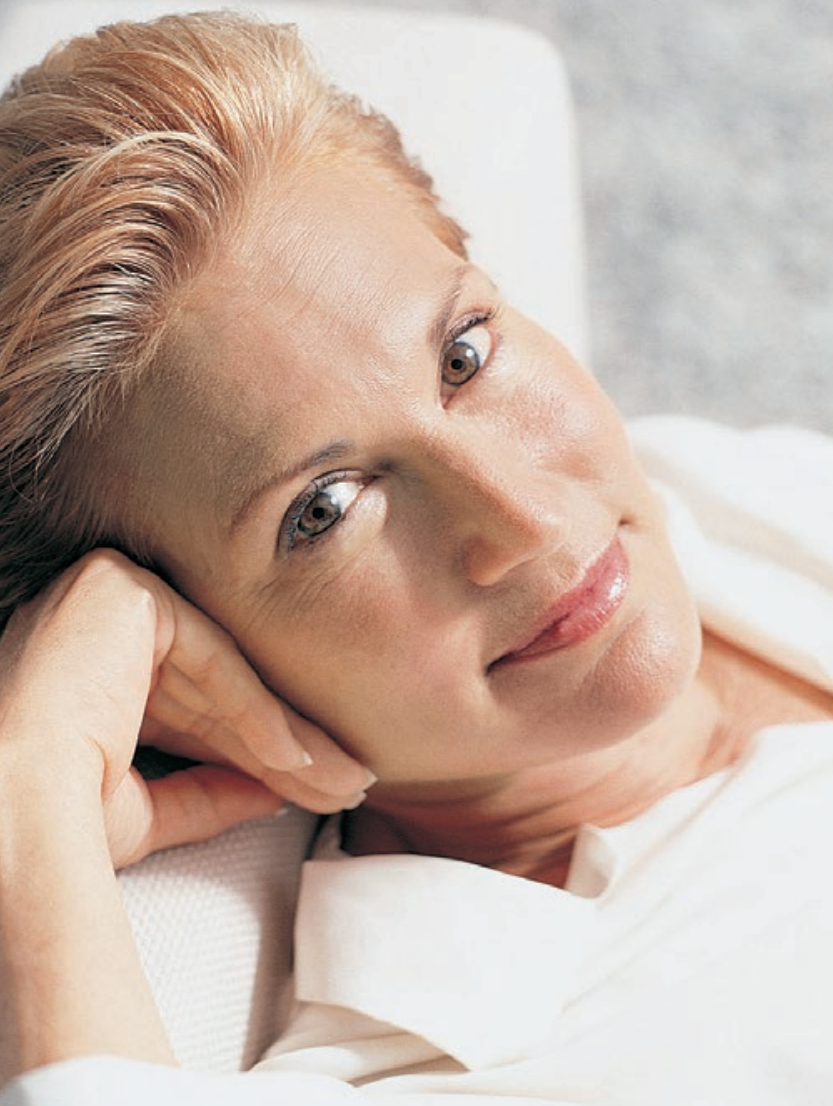

Bundesverband für

Gesundheitsinformation und Verbraucherschutz - Info Gesundheit e.V.

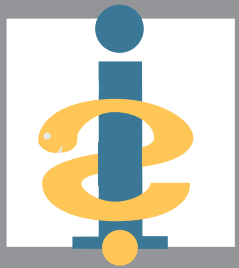

\title{
BLASENSCHWÄCHE BEI FRAUEN
}


2. BLASENSCHWÄCHE BEI FRAUEN 4

Ursachen und Risikofaktoren 4

Die häufigsten Formen von Inkontinenz bei Frauen

3. DAS MACHT IHR ARZT 8

Untersuchungen 8

Behandlungsmethoden 9

Belastungsinkontinenz 10

Dranginkontinenz 12

4. DAS KÖNNEN SIE TUN! 14

I0 Tipps zur Vorbeugung und Linderung von Blasenschwäche

5. ÜBUNGEN ZUR KRÄFTIGUNG DES BECKENBODENS 
Unsere Blase ist ein äußerst leistungsfähiges Organ. Als Behälter für die Zwischenlagerung von Urin ist sie dehnbar wie ein Luftballon und besitzt ein Fassungsvermögen von bis zu einem Liter. Ohne Blase müssten wir ununterbrochen Urin abgeben, da unsere Nieren laufend Harn produzieren. Doch zum Glück sammelt die Blase den Urin über mehrere Stunden und meldet erst bei einem Füllvolumen von 300 bis 600 Millilitern starken Harndrang.

Bis dahin sorgt ein spezielles Verschlusssystem mit Unterstützung der Beckenbodenmuskulatur dafür, dass der Urin zurückgehalten wird. Den Zeitpunkt der Blasenentleerung steuern wir bewusst. Gehen wir zur Toilette, leiten dieselben Muskeln, die die Blase in der Sammelphase verschließen, den Urin nun in die Harnröhre. Während der Blasenverschluss erschlafft, zieht sich die Muskulatur zusammen und treibt den Urin nach draußen.

An der Koordination dieser komplizierten Abläufe sind

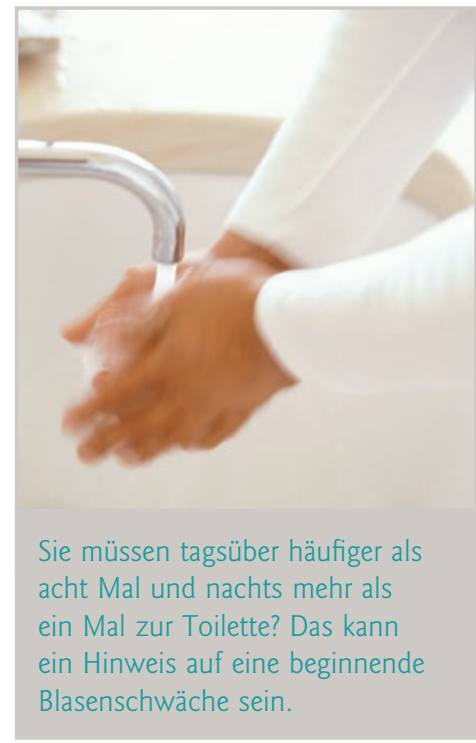
jedoch nicht nur Blasenmuskel sowie innere und äußere Schließmuskeln beteiligt, sondern auch alle drei Komponenten des Nervensystems. Ist dieser Kreislauf an

\section{EFIN NTION Als Blasenschwäche oder Harninkon-} tinenz wird der unfreiwillige Verlust von Urin bezeichnet. Die Betroffenen können den Zeitpunkt der Blasenentleerung nicht mehr willentlich bestimmen und verlieren die Fähigkeit, Urin zurückzuhalten. 
NERVENSYSTEM Das vegetative Nervensystem besteht aus Sympathikus und Parasympathikus, die völlig gegensätzlich auf den Organismus wirken und sich gerade deshalb hervorragend ergänzen. Während der Sympathikus unseren Körper in einen Zustand höherer Aufmerksamkeit und Leistungsfähigkeit versetzt, sorgt der Parasympathikus als sein Gegenspieler für Regeneration, Ruhe und Entspannung. In Bezug auf unsere Blasentätigkeit bedeutet das vereinfacht gesagt, dass der Sympathikus für die Speicherfunktion (= Anspannung der Muskulatur) und der Parasympathikus für die Entleerung der Blase (= Entspannung der Muskulatur) zuständig ist. Neben dem vegetativen Nervensystem, das wir nicht beeinflussen können, erfolgt die bewusste An- und Entspannung der Muskulatur und damit letztlich der Vorgang des Wasserlassens über das willkürliche Nervensystem.

einer Stelle gestört, kann dies zu einer Blasenschwäche führen. Je nachdem, welcher Teil betroffen ist, kommt es zu unterschiedlichen Formen von Harninkontinenz.

\section{BLASENSCHWÄCHE BEI FRAUEN}

\section{口 URSACHEN UND RISIKOFAKTOREN}

Blasenschwäche kann verschiedene Ursachen haben. Schon junge Frauen sind überdurchschnittlich häufig betroffen, wobei das Risiko mit zunehmendem Alter noch steigt: Schätzungen zufolge leiden 40 Prozent aller Frauen über 60 Jahre an Blasenschwäche. In den meisten Fällen lässt sich keine auslösende Erkrankung wie ein Tumor, eine Rückenmarksverletzung, multiple Sklerose, Parkinson oder ein schwerer Bandscheibenvorfall finden. Die Gründe liegen vielmehr in der Natur der Frau. Die Stütz- und Haltefunktion des weiblichen Beckenbodens ist sehr viel stärker beansprucht, weil Frauen ein breiteres Becken haben als Männer. 
Das ist notwendig, um Kinder auf die Welt zu bringen. Damit sich die Gebärmutter während der Schwangerschaft ausdehnen kann, hat sie nach oben hin Spielraum und wird nur von unten durch die Beckenbodenmuskulatur getragen. Ohne Beckenboden hätten die Organe im Bauchraum keinen Halt. Doch Geburten, häufige Unterleibsoperationen, Übergewicht oder körperliche Belastungen können den Beckenboden auf Dauer schwächen.

ZA H LEN In Deutschland gibt es rund sechs Millionen Menschen, deren Blasenfunktion gestört ist. Durch anatomische und hormonelle Unterschiede sind Frauen schon in jungen Jahren doppelt so häufig betroffen wie Männer.

Das wiederum kann zu einer Absenkung der Gebärmutter und anderer Beckenorgane führen sowie den Verschlussmechanismus der Blase beeinträchtigen. Besonders gefährdet sind Frauen mit einer Bindegewebsschwäche.

In diesen Fällen macht sich die Blasenschwäche bereits frühzeitig bemerkbar, während sich bei den meisten Frauen die Beschwerden erst nach der Menopause einstellen. Durch die hormonellen Veränderungen in den Wechseljahren produziert der weibliche Körper weniger Östrogen. Dadurch verliert das Gewebe zusätzlich an Elastizität und wird schwächer. Ebenfalls bedingt durch den Östrogenmangel werden die Schleimhäute

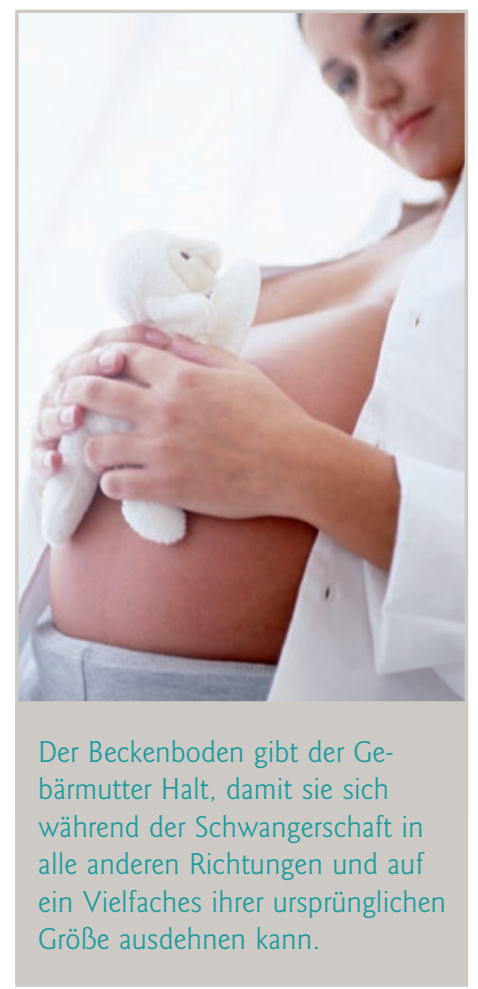




\section{URSACHEN UND RISIKOFAKTOREN}

- Häufige Harnwegsinfektionen und Blasenentzündungen

- Schwangerschaft und Geburt

- Unterleibsoperationen

- Schwäche der Beckenbodenmuskulatur

- Schwäche des Bindegewebes

- Senkung von Gebärmutter und Beckenboden

- Wechseljahre und Östrogenmangel

im Genitalbereich schlechter durchblutet, wodurch der pH-Wert der Scheide ansteigen kann. Das wiederum kann das Eindringen von Keimen und Krankheitserregern erleichtern und in der Folge zu vermehrten Blasenentzündungen und Harnwegsinfektionen führen. Da Frauen eine kürzere Harnröhre haben als Männer, die zudem in unmittelbarer Nähe des Darmausgangs liegt, sind sie ohnehin anfälliger für Infekte und Entzündungen. All das belastet die Blase.

\section{ZAA H LEN Etwa 25 Prozent aller Frauen zwischen 25} und 35 Jahren leiden vorübergehend an Blasenschwäche als Folge von Entbindungen.

\section{口 DIE HÄUFIGSTEN FORMEN VON INKONTINENZ BEI FRAUEN}

Frauen, die unter einer schwachen Blase leiden, sind besonders häufig von Belastungsinkontinenz betroffen. Bei körperlicher Anstrengung wie zum Beispiel Husten, Lachen, Niesen, Heben von schweren Lasten oder Treppensteigen kommt es zu ungewolltem Harnverlust, ohne dass die Betroffenen zuvor einen Harndrang verspüren. Ursache ist eine Schwäche der Beckenbodenmuskulatur, 
die dazu führt, dass der Schließmechanismus der Harnröhre nicht mehr zuverlässig arbeitet.

Bei der Dranginkontinenz ist der Verschlussapparat der Harnblase zwar intakt, die Blasenmuskulatur jedoch extrem verspannt, was zu einer Überreaktion führt (siehe unten stehenden Kasten). Die Betroffenen verspüren bei dieser Form der Blasenschwäche einen so starken und plötzlichen Harndrang, dass sie es nicht mehr bis zur Toilette schaffen. Dranginkontinenz kann bei Frauen durch häufige Harnwegsentzündungen ausgelöst werden. Sehr

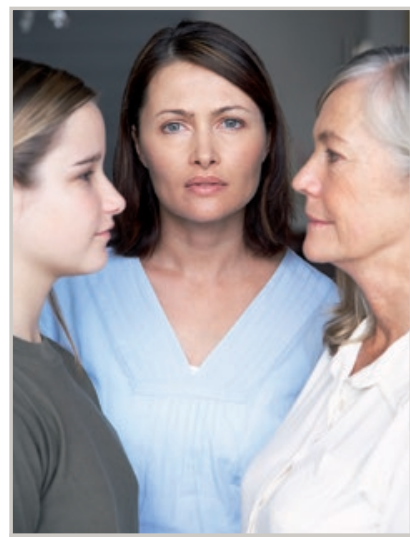

Blasenschwäche kann Frauen in jedem Alter treffen.

Schieben Sie den Arztbesuch nicht auf, wenn Sie die ersten Symptome bemerken! häufig kommen auch Mischformen der Drang- und Belastungsinkontinenz vor, die jedoch ebenso gut zu behandeln sind wie die beiden Hauptformen selbst.

\section{FORMEN VON INKONTINENZ}

- Belastungsinkontinenz: ungewollter Urinverlust bei körperlicher Anstrengung

- Dranginkontinenz: gestörter Informationsaustausch zwischen Gehirn und Blase

- sensorische Dranginkontinenz (überempfindliche Blase): Vortäuschen einer übervollen Blase

- motorische Dranginkontinenz (überaktive Blase): nicht beeinflussbares Zusammenziehen des Blasenmuskels

- Reflexinkontinenz: unfreiwilliger Urinverlust ohne Harndrang durch unkontrollierbare Nervenreflexe

- Überlaufinkontinenz: ständige Abgabe kleiner Urinmengen ohne vollständige Blasenentleerung 
3. DAS MACHT IHR ARZT

\section{UNTERSUCHUNGEN}

Das Wichtigste vorweg: Damit Ihnen geholfen werden kann, müssen Sie aktiv werden. Überwinden Sie Angst und Scham und vertrauen Sie sich Ihrem Arzt oder Ihrer Ärztin an. Sie sind mit Ihrem Problem nicht alleine! Blasenschwäche ist gut behandelbar und kein Schicksal, das Sie hinnehmen müssen. Erster Ansprechpartner sollte der Hausarzt, Ihr

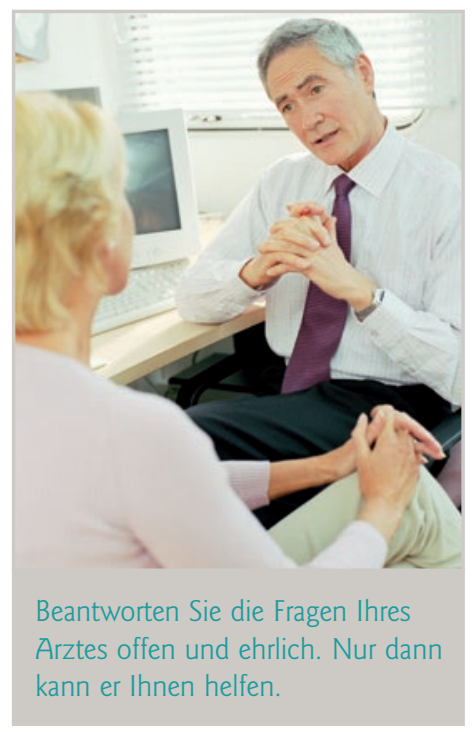

Frauenarzt oder ein Urologe sein. Er wird Ihnen viele Fragen zur Art der Beschwerden und der medizinischen Vorgeschichte stellen. Da dieses Gespräch ein wichtiger Bestandteil des Diagnoseverfahrens ist, sollten Sie sich gut darauf vorbereiten (siehe Kasten).

Parallel dazu wird Ihr Arzt eine körperliche Grunduntersuchung vornehmen. Durch Abtasten des Bauchraums und der Nierengegend erhält er erste Hinweise auf krankhafte Veränderungen von Nieren, Blase oder Harnleiter. Zur Beurteilung der Beckenbodenmuskulatur und der Lage der Blase werden Frauen zudem gynäkologisch untersucht. Dabei kann Ihr Frauenarzt bzw. Ihre Frauenärztin auch feststellen, ob ein Hormonmangel vorliegt. Bei Verdacht auf eine Harnwegsinfektion prüft das Labor, ob sich Bakterien im Urin nachweisen lassen. Sollten die bisherigen Ergebnisse nicht ausreichen, um eine gesicherte Diagnose zu stellen, schließen sich weitere Untersuchungen an. Mit Hilfe von Ultraschall und Röntgen kann sich Ihr Arzt einen Überblick über Lage, Form und Beschaffenheit der betreffenden Organe verschaffen. Die urodynamische Untersuchung vermittelt einen Eindruck über den Ablauf der Blasentätigkeit. 
DER A RZT B ESUCH Ihr Arzt wird Ihnen folgende Fragen stellen. Es ist hilfreich, wenn Sie sich vorab Notizen zu den Fragen machen.

- Seit wann leiden Sie unter Blasenschwäche?

- Wie häufig müssen Sie zur Toilette?

- Wie oft verlieren Sie Urin, ohne es zu wollen, und wie viel? In welchen Situationen geschieht das?

- Spüren Sie oft einen starken Harndrang? Müssen Sie diesem sofort nachgeben? Oder können Sie den Gang zur Toilette einige Zeit hinauszögern?

- Haben Sie Schmerzen beim Wasserlassen?

- Ist der Harnstrahl kräftig oder abgeschwächt, oder geht der Harn sogar nur tröpfelnd ab?

- Haben Sie das Gefühl, Ihre Blase vollständig entleeren zu können?

- Wie lange dauert es, bis die Blase entleert ist?

- Haben Sie Kinder geboren? Wenn ja, wie viele? Sind die Geburten normal verlaufen oder gab es Komplikationen?

- Befinden Sie sich in den Wechseljahren?

- Leiden Sie häufig unter Blasenentzündungen?

- Verwenden Sie Inkontinenzhilfsmittel?

- Liegen andere Erkrankungen vor?

- Welche Medikamente nehmen Sie ein?

\section{BEHANDLUNGSMETHODEN}

Die genaue Diagnose ist deshalb so wichtig, weil die Therapie entscheidend von der Form der Blasenschwäche abhängt. Deshalb gilt es zunächst einmal, bestimmte Grunderkrankungen auszuschließen oder entsprechend zu behandeln. In den meisten Fällen finden sich für die Symptome jedoch keine ernsthaften Ursachen. Bei der Behandlung von Belastungs- und Dranginkontinenz gibt es eine Reihe erfolgreicher Therapiemöglichkeiten, die wir Ihnen im Folgenden vorstellen. 


\section{Belastungsinkontinenz}

Physikalische Therapie: Die häufigste Ursache für Belastungsinkontinenz bei Frauen ist ein schwacher Beckenboden. Trifft das auch in Ihrem Fall zu, wird Ihnen Ihr Arzt ein entsprechendes Trainingsprogramm verordnen. Erfahrene Physiotherapeuten zeigen Ihnen die richtigen Übungen zur Stärkung der Muskulatur, so dass Sie nach einiger Zeit in der Lage sind, das Training auch zu Hause durchzuführen. Je häufiger Sie die Übungen machen und dafür zusätzliche Hilfsmittel wie

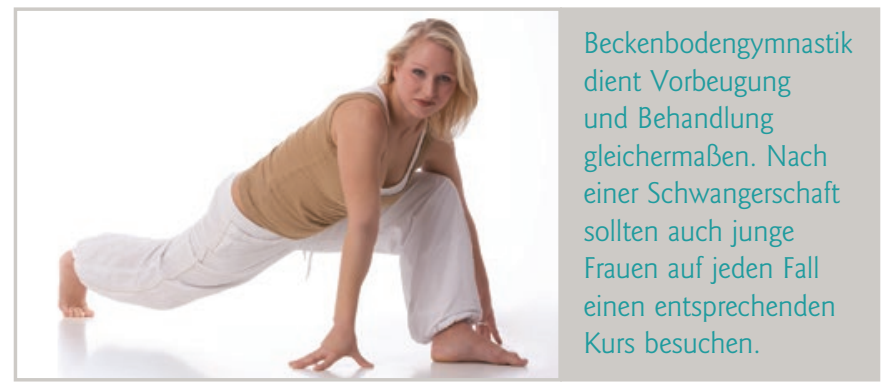

\section{BIOFEEDBACK UND}

FLERTROSTIMULATION Da viele Patientinnen trotz fachkundiger Anleitung Schwierigkeiten haben, die Beckenbodenmuskulatur zu spüren und willentlich anzuspannen, dient das sogenannte Biofeedback-Verfahren der Kontrolle. Dabei werden die Muskelspannungen über einen Sensor in der Scheide auf einem Monitor grafisch sichtbar gemacht. So können Sie am Bildschirm verfolgen, ob Sie die Übung korrekt ausführen oder nicht. Tritt nach drei Monaten keine Besserung ein, empfiehlt sich die zusätzliche Stimulation des Beckenbodens mit Elektroimpulsen. Dabei werden spezielle Elektroden in die Scheide eingeführt, die mit Hilfe von Reizstrom die Muskulatur zur Kontraktion bringen. 
Vaginalkegel verwenden, desto schneller wird Ihre Blase bei körperlicher Belastung wieder dicht verschließen. Um ein Gefühl dafür zu bekommen, welche Muskeln genau gemeint sind, sollten Sie beim Wasserlassen häufiger einmal Ihren Urinstrahl unterbrechen. Dabei werden sehr viele Muskeln des Beckenbodens aktiviert - und gleichzeitig trainiert.

\section{Medikamentöse Behandllung:}

Zur Behandlung von Blasenschwäche, die durch einen Hormonmangel in den Wechseljahren hervorgerufen wurde, können Östrogene in Form von Salben oder Vaginalzäpfchen verabreicht werden. Mittel wie OeKolp ${ }^{\circledR}$, Ortho-Gynest ${ }^{\circledR}$, Ovestin ${ }^{\circledR}$ oder $\mathrm{Xapro}^{\oplus}$ halten das Gewebe elastisch und sorgen für eine bessere Durchblutung der Blase.

\section{Operative Verfahren: Stellt} sich trotz all dieser Maßnahmen kein Erfolg ein, kann es sein, dass Ihnen Ihr Arzt zu einer Operation rät. Je nach Ursache der Blasenschwäche kommen unterschiedliche Verfahren in Betracht. So besteht zum Beispiel die Möglichkeit, das Bindegewebe des Beckenbodens zu straffen. Ist ein Absenken der Blasenorgane für die Inkontinenz verantwortlich, können Blase und Harnröhre angehoben werden, um die ursprüngliche Lage wiederherzustellen. Bei einer Harnröhrenunterspritzung werden Kunststoffe oder natürliche Bindegewebssubstanzen um die Harnröhre im Bereich des Blasenhalses gespritzt. Dieser zusätzliche Druck verbessert die Verschlussfähigkeit der Harnblase. Die sogenannte TVT-Operation (Tension-free Vaginal Tape) lässt sich sogar bei örtlicher Betäubung vornehmen. 


\section{Dranginkontinenz}

\section{Physikalische Therapie:}

Die Überaktivität der Blasenmuskulatur kann verschiedene Ursachen haben - etwa chronische Blasenentzündungen, Blasensteine, Nervenerkrankungen und Stoffwechselstörungen, die natürlich entsprechend behandelt werden müssen. In den

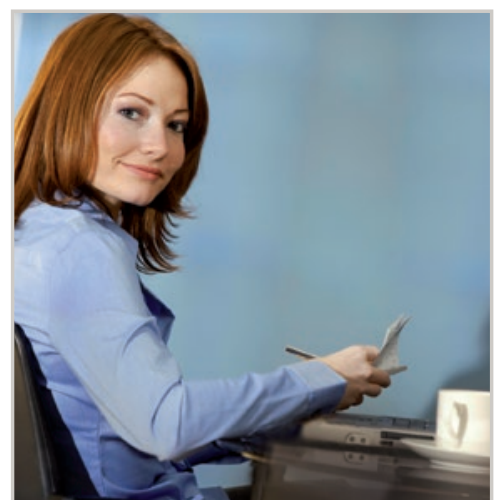

Viel trinken und trotzdem den Harndrang hinauszögern - das Blasentraining erfordert viel Disziplin. Halten Sie die Fortschritte schriftlich fest. meisten Fällen ist die Dranginkontinenz jedoch nicht auf eine organische Störung zurückzuführen. Hier geht es in erster Linie darum, die Symptome zu bekämpfen. Als Hilfe zur Selbsthilfe sollten Sie mit einem gezielten Blasen- und Toilettentraining die Kontrolle über Ihre Blase verbessern und die Abstände zwischen den Toilettengängen schrittweise verlängern. So wird die Blase wieder an größere Füllmengen gewöhnt. Die Ergebnisse tragen Sie am besten in ein Miktionstagebuch (Miktion $=$ Blasenentleerung) ein. Notieren Sie außerdem, wann Sie Harndrang verspüren, nach wie viel Minuten Sie diesem nachgeben, ob Sie unfreiwillig Urin verloren haben und wie viel Sie trinken. Ergänzend dazu empfiehlt sich auch bei Dranginkontinenz ein Beckenbodentraining zur Kräftigung der Muskulatur.

Medikamentöse Behandlung: Parallel zum Blasentraining gehört die medikamentöse Behandlung zur Standardtherapie bei Dranginkontinenz. Die Medikamente sorgen für eine Entspannung der Blasenmuskulatur, die dadurch wieder mehr Urin speichern kann. Dabei kommen vor allem Anticholinergika zum Einsatz, die in den meisten Fällen eine Linderung der Symptome bewirken. Zu den möglichen Nebenwirkungen von Anticholinergika gehören Mundtrockenheit, Magen-Darm-Störungen und trockene Haut. 
Anticholinerge Wirkstoffe: Anticholinerge Wirkstoffe sind u. a. Darifenacin $\left(\right.$ Emselex $^{\circledR}$ ), Oxybutynin (Dridase ${ }^{\circledR} /$ Generika, Kentera ${ }^{\circledR}$ ), Propiverin $\left(\right.$ Mictonorm $\left.^{\circledR}\right)$, Solifenacin $\left(\right.$ Vesikur $\left.^{\circledR}\right)$, Tolterodin (Detrusitol ${ }^{\circledR}$ ), Fesoterodin $\left(\right.$ Toviaz $\left.^{\circledR}\right)$, Trospiumchlorid $\left(\right.$ Spasmex $\left.^{\circledast}\right)$.

2009 führte der BGV e.V. eine Befragung zur Behandlung der Harninkontinenz bei Frauen bei niedergelassenen Gynäkologen durch. Bei der Medikation zur Dranginkontinenz benannten die Ärzte die Wirkstoffe Solifenacin $\left(\right.$ Vesikur $^{\circledR}$ ) und Trospiumchlorid (Spasmex ${ }^{\circledast}$ ) als die am häufigsten verordneten Präparate. Als sicherste Wirkstoffe mit den geringsten beobachteten Nebenwirkungen wurden Trospiumchlorid (Spasmex ${ }^{\circledR}$ ), Solifenacin $\left(\right.$ Vesikur $\left.^{\circledR}\right)$ und Tolterodin $\left(\right.$ Detrusitol $\left.^{\circledR}\right)$ eingeschätzt. Die ausführlichen Ergebnisse der Umfrage können Sie im Internet unter www.frauen-blasenschwaeche.de abrufen.

Bei leichten Formen von Dranginkontinenz können auch pflanzliche Mittel mit Kürbis (z. B. Cysto-Urgenin ${ }^{\circledR}$, Granu Fink ${ }^{\circledR}$ ), Goldrute (z. B. Inconturina ${ }^{\circledR}$ ) oder Cranberry (z. B. Cranberola ${ }^{\circledR}$ ) helfen - zumindest lindern sie die Reizzustände. Antibiotika kommen nur dann zum Einsatz, wenn Harnwegsinfekte oder häufige Blasenentzündungen als Ursache der Drangsymptomatik diagnostiziert wurden.

Operative Verfahren: Da der Verschlussapparat der Harnblase bei der Dranginkontinenz intakt ist, kann eine Operation hier nichts ausrichten. Es sei denn, Blasensteine sind für die Symptome verantwortlich, dann müssen diese natürlich entfernt werden.

UNSER TIPP Unabhängig davon, unter welcher Form von Harninkontinenz Sie leiden - wichtig ist, dass Sie die Therapieempfehlungen Ihres Arztes konsequent befolgen und auch selbst aktiv werden. So gewinnen Sie wertvolle Lebensqualität zurück. 


\section{DAS KÖNNEN SIE TUN!}

I 10 TIPPS ZUR VORBEUGUNG UND LINDERUNG VON BLASENSCHWÄCHE

1. Stärken Sie Ihre Beckenbodenmuskulatur. Praktische Übungen finden Sie im nächsten Kapitel.

2. Reduzieren Sie Ihr Gewicht. Überflüssige Pfunde belasten den Beckenboden.

3. Achten Sie auf die richtige Körperhaltung. Alle Bewegungen und Positionen, die den Rücken schonen, sind auch gut für den Beckenboden. Halten Sie sich aufrecht und tragen Sie schwere Gegenstände stets nah am Körper.

4. Treiben Sie regelmäßig Sport. Bewegung fördert die Durchblutung, kräftigt die Muskulatur - das kommt auch Ihrer Blase zugute. Vermeiden Sie Sportarten, die den Beckenboden belasten (Aerobic, Joggen) und wählen Sie einen Sport, der den Beckenboden entlastet (Schwimmen, Yoga, Gymnastik, Walking).

5. Sorgen Sie für einen leichten Stuhlgang. Übermäßiges Pressen führt zu einer Überlastung der Beckenbodenmuskulatur. Ernähren Sie sich deshalb ballaststoffreich und trinken Sie morgens ein Glas Wasser auf nüchternen Magen.

6. Trinken Sie viel ( 1,5 bis 2 Liter pro Tag), um sich vor Blasensteinen und Infektionen zu schützen. Auf harntreibende Getränke wie Kaffee und Alkohol sollten Sie hingegen verzichten. Vermeiden Sie es, kurz vor dem Schlafengehen zu trinken, um nachts nicht ständig zur Toilette zu müssen.

7. Beugen Sie Harnwegsinfektionen vor, da diese das Inkontinenzrisiko erhöhen. Halten Sie sich warm und achten Sie darauf, dass keine Bakterien aus dem Darm in die Scheide gelangen. Nach dem Geschlechtsverkehr sollten Sie umgehend zur Toilette gehen, um Keime möglichst schnell hinauszuspülen. 
Sitzen Sie beim Wasserlassen immer aufrecht, damit die Blase vollständig entleert werden kann.

8. Trainieren Sie Ihre Blase. So verbessern Sie bei Dranginkontinenz die Kontrolle über Ihre Blase und vergrößern die Abstände zwischen den Toilettengängen. Vor dem Training und währenddessen ist es hilfreich, ein Miktionstagebuch zu führen.

9. Sorgen Sie für Entspannung. Bei häufigem Harndrang können Techniken wie Yoga, autogenes Training oder progressive Muskelentspannung helfen, die Symptome stressbedingter Ursachen von Blasenschwäche zu lindern.

10. Bleiben Sie gesund und rauchen Sie nicht! Chronischer Husten kann auf Dauer zu einer Schwächung der Beckenbodenmuskulatur beitragen und Harninkontinenz verursachen.

\section{INFORMATIONEN IMINTERNET}

- www.frauen-blasenschwaeche.de Bundesverband für Gesundheitsinformation und Verbraucherschutz - Info Gesundheit e.V., Heilsbachstraße 32, 53 I23 Bonn, E-Mail: info@bgv-info-gesundheit.de

- www.kontinenz-gesellschaft.de Deutsche Kontinenz Gesellschaft e.V., Friedrichstraße 15, 60323 Frankfurt a. M., Tel. 069/795 88 393, Fax 069/795 88383

- www.medizin-netz.de/krankheiten/harninkontinenz/ Medizinischer Informationsdienst im Internet

- www.dieblase.de Auf dieser Internetseite finden Sie umfangreiche Informationen zu Blasenschwäche, praktische Übungen und Tipps zum Erhalt der Lebensqualität. In einem Online-Lexikon können Sie die wichtigsten Begriffe von $A$ bis $Z$ nachschlagen. Mediziner beantworten Ihre Fragen in einem Experten-Forum und -blog. Das Download-Angebot umfasst u. a. ein Miktionstagebuch, Checklisten zur Vorbereitung auf den Arztbesuch und einen Patientenratgeber.

- www.medikamente-im-alter.de Ältere Menschen reagieren im allgemeinen empfindlicher auf Arzneimittel mit anticholingergen Effekten. Wissenschaftler haben auf der „Priscus-Liste“ Wirkstoffe aufgelistet, die für ältere Menschen bedenklich sein können. Die Internetseite bietet eine Übersicht und führt Therapiealternativen auf. 
5. ÜBUNGEN ZUR KRÄFTIGUNG DES BECKENBODENS

Unsere Beckenbodenmuskulatur lässt sich ebenso trainieren wie die Muskeln der Arme, Beine oder des Bauchs. Wir können sie anspannen und danach wieder lockern. Je häufiger Sie die Übungen durchführen, desto schneller gewinnen Sie die Kontrolle über Ihre Blase zurück. Bauen Sie die Gymnastik in Ihren Tagesablauf ein, je nach persönlicher Vorliebe morgens nach dem Aufstehen oder abends vor dem Zubettgehen. Auch zwischendurch können Sie immer mal wieder etwas für Ihren Beckenboden tun, indem Sie die Muskeln beim Sitzen in der Bahn oder im Büro fünfmal hintereinander anspannen und wieder lockern. Oder Sie unterbrechen beim Wasserlassen mehrmals den Urinstrahl. Für die folgenden Übungen benötigen Sie lediglich eine weiche Unterlage, bequeme Kleidung und einen Stuhl - schon kann's losgehen! Wir wünschen Ihnen viel Spaß und vor allem viel Erfolg!

\section{ÜB UNG I}

Legen Sie sich auf den Rücken und stellen Sie die Beine schulterbreit auf. Heben Sie den Po so weit an, bis Oberschenkel und Oberkörper eine Linie bilden. Bei dieser Bewegung werden auch Bauch- und Rückenmuskeln beansprucht. Das Gewicht ruht auf den Schulterblättern. Heben Sie die Füße nun viermal im Wechsel wenige Zentimeter vom Boden. Achten Sie darauf, das Becken waagerecht zu halten.

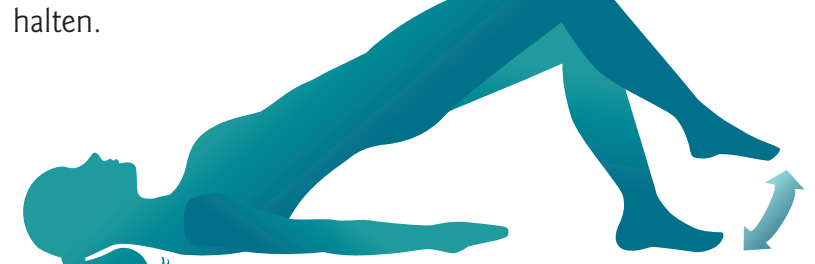

$3 \times 4$ Wiederholungen 

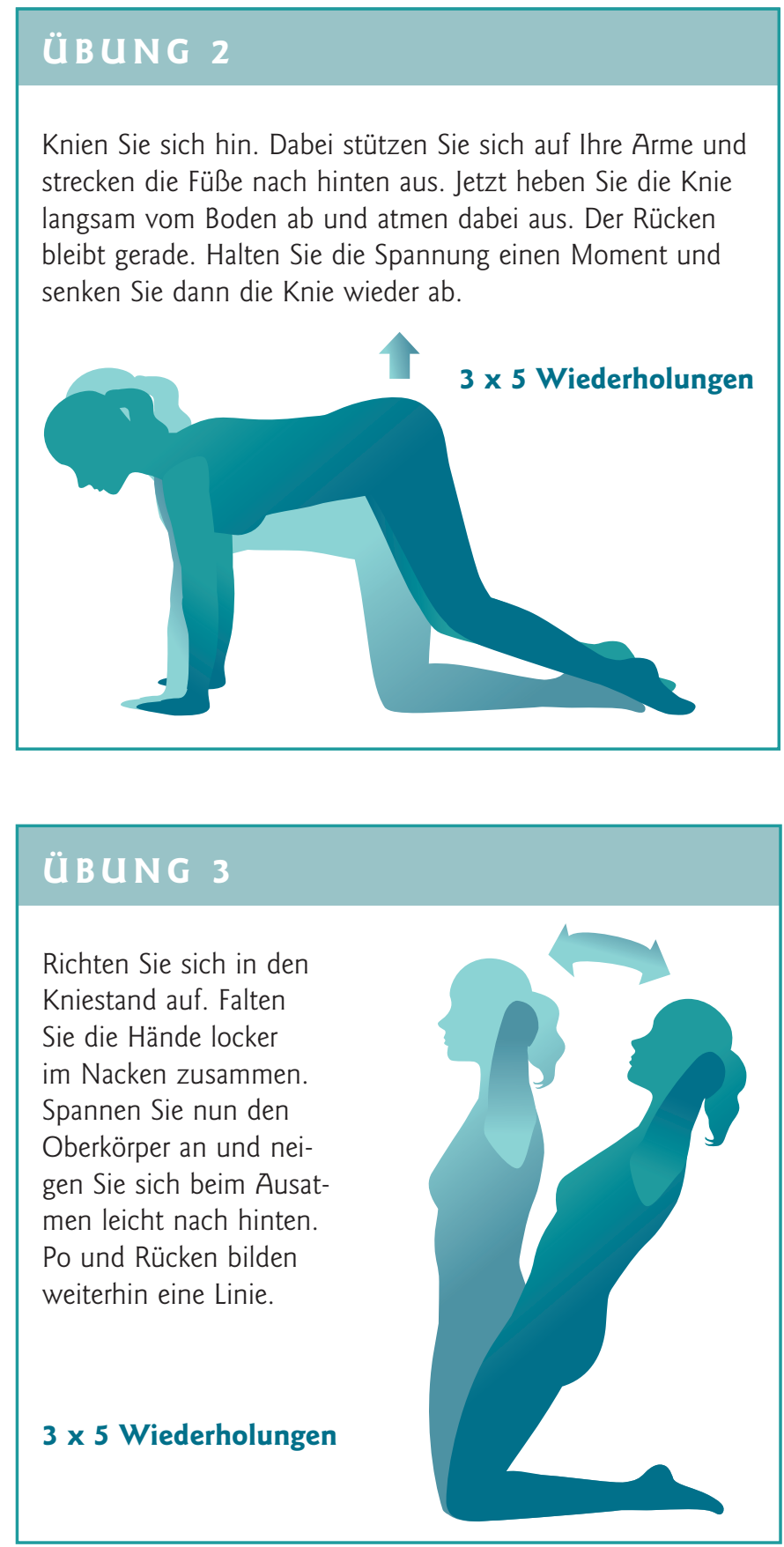


\section{ÜBUNG 4}

Legen Sie sich auf die Seite, der untere Arm liegt unter dem Kopf, der obere ist vor der Brust aufgestellt, die Füße liegen aufeinander. Drücken Sie nun die Fersen fest aufeinander und spreizen Sie das obere Knie. Dabei atmen Sie aus, halten die Position einen Moment und schließen die Knie beim Einatmen wieder.

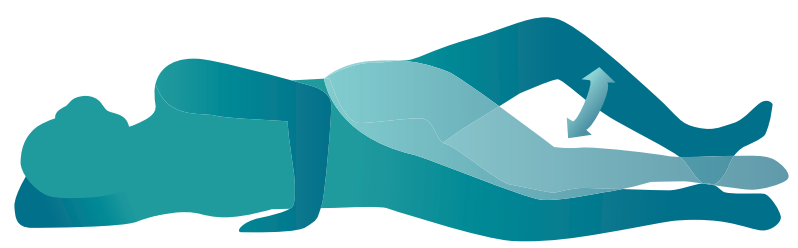

$3 \times 5$ Wiederholungen

\section{ÜBUNG 5}

Setzen Sie sich aufrecht und mit leicht gespreizten Beinen auf einen Stuhl. Umfassen Sie Ihr rechtes Knie mit beiden Händen, ziehen Sie es leicht an den Körper heran und halten Sie diese Stellung für einige Sekunden. Dann lassen Sie das Knie plötzlich los und recken beide Arme empor. Danach führen Sie die Übung mit dem linken Knie durch.

je 5 Wiederholungen

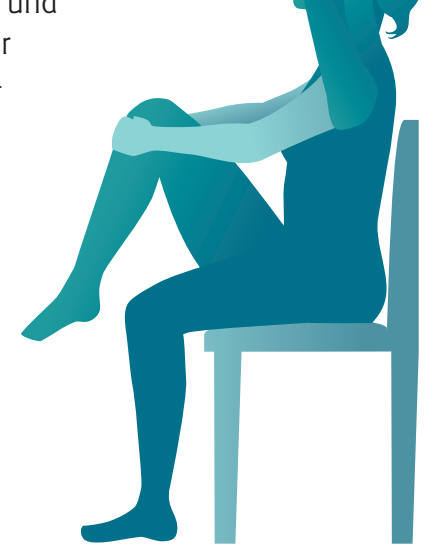




\section{Information Aufklårung dank Ihrer Unterstützung!}

Gesundheit ist unser kostbarstes Gut. Doch trotz des medizinischen Fortschritts steigt die Zahl chronischer und anderer Erkrankungen. Der Bundesverband für Gesundheitsinformation und Verbraucherschutz - Info Gesundheit e.V. (BGV) hat sich zum Ziel gesetzt, dem Informationsbedürfnis von Patienten, Verbrauchern, Ärzten, Apothekern und anderen Beschäftigten aus dem medizinischen Bereich nachzukommen. Dabei sind wir auf Ihre Mithilfe angewiesen, da wir keine öffentlichen Mittel beanspruchen.

Bitte unterstützen Sie unsere Arbeit. Mitgliedsbeiträge und Spenden sind steuerlich absetzbar. Bis 200 Euro gilt die abgestempelte Quittung bzw. Kopie des Bankauszugs als Spendenbescheinigung. Für Spenden über 200 Euro senden wir Ihnen die Spendenbescheinigung gerne auf Anfrage zu. Vielen Dank!
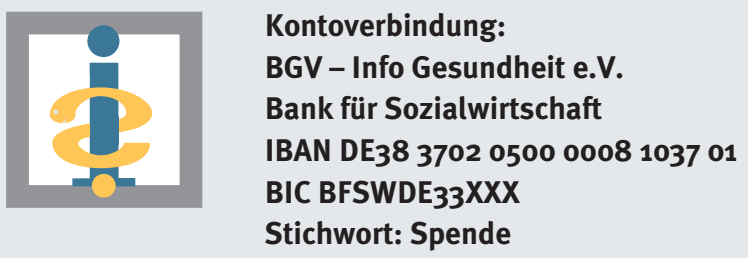

Kontoverbindung: BGV - Info Gesundheit e.V. Bank für Sozialwirtschaft IBAN DE38 370205000008103701 BIC BFSWDE 33 XXX

Stichwort: Spende

Für Spenden können Sie auch unser Online-Formular nutzen: www.bgv-info-gesundheit.de/spende.html

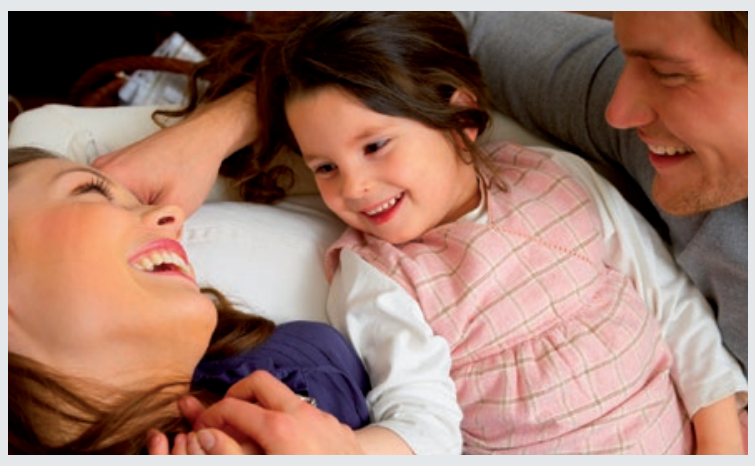




\section{Bildnachweis:}

Digital Vision/Prime Time: Seite I

Imageshop/Bathroom Rituals: Seite 3

Imageshop/Being pregnant: Seiten 5 und II

Fancy/Family Bonds: Seite 7

Bananastock/Family Health: Seite 8

Fotolia/Franz Pfluegl: Seite 10

Comstock/Lifestyles at Home: Seite 12

Fotolia/Yuri Arcurs: Seite 19

\section{Illustrationen Katharina Heidman:}

Seiten 16 bis 18

Auflage 2017

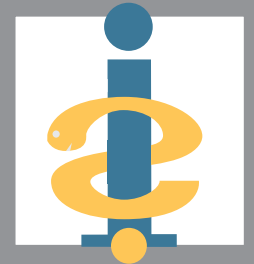

Bundesverband für Gesundheitsinformation und

Verbraucherschutz - Info Gesundheit e.V.

Geschäftsführer: RA Erhard Hackler

Heilsbachstraße 32, 53123 Bonn

Telefon: 0228/9379950

www.bgv-info-gesundheit.de

\section{(C) Bundesverband für} Gesundheitsinformation und Verbraucherschutz - Info Gesundheit e. V., Referat Presseund Öffentlichkeitsarbeit, Heilsbachstraße 32, 53123 Bonn Telefon: 0228/9379950 Telefax: 0228/3679390

Abdruck, auch auszugsweise, nur mit Genehmigung des Herausgebers. 